\title{
Mitral valve repair rates in degenerative mitral valve disease correlate with surgeon and hospital procedural volume
}

\author{
Stephanie Wayne ${ }^{1}$, Catherine Martin² ${ }^{2}$ Julian Smith $^{1}$, and Aubrey Almeida ${ }^{1}$ \\ ${ }^{1}$ Monash Health \\ ${ }^{2}$ Monash University
}

August 14, 2020

\begin{abstract}
Study aim: To determine the relationship between surgeon and hospital procedural volume, and mitral valve repair rates and 30day mortality for degenerative mitral regurgitation (MR), in Australian cardiac surgical centres. Methods: 4,970 patients who underwent surgery for degenerative MR between January 2008 and December 2017 in the Australian and New Zealand Society of Cardiac and Thoracic Surgeons (ANZSCTS) Database were retrospectively included. Univariate and multivariate regression analyses examined surgeon and hospital procedural volumes for associations with repair rate and mortality. Results: Repair rates varied widely by caseload; from $56.7 \%$ to $80.4 \%$ for lowest to highest volume surgeons; and from $52.0 \%$ to $76.1 \%$ for lowest to highest volume hospitals. Compared to surgeons performing [?]5 procedures/annum, surgeons performing 10.1-20/annum were more likely to repair the valve (OR 2.91, 95\% Confidence Interval [CI] 1.50-5.64, p=0.002), particularly if performing $>20$ /annum (OR 3.9, 95\% CI 1.62-9.37, $\mathrm{p}=0.002$ ). Compared to hospitals performing [?]10/annum, those performing any number of procedures $>10$ demonstrated increased likelihood of repair (caseload 10.1-20/year OR 2.04, 95\% CI 1.30-3.20, $\mathrm{p}=0.002)$ though odds did not increase above this threshold. Low incidence of 30-day mortality $(83$ of $4,964,1.67 \%)$ limited analysis of contributing variables; procedural volume did not confer a survival benefit, though mortality rates were lowest for highest volume proceduralists and hospitals. Conclusions: Surgeon and hospital caseload were significantly associated with repair rates of degenerative MR. A threshold minimum of 10 procedures annually for surgeons and hospitals should be utilised to maximise repair rates, and ideally of 20 for surgeons. Mortality was low and may not be significantly impacted by procedural volume.
\end{abstract}

\section{Introduction}

Degenerative mitral valve disease is the most common valvular pathology worldwide, affecting up to $10 \%$ of adults aged 75 years and over [1]. The Valvular Heart Disease Guidelines of both the European Society of Cardiology (ESC)/European Association for Cardio-Thoracic Surgery (EACTS) and the American College of Cardiology (ACC)/American Heart Association (AHA) recommend that mitral valve repair as the preferred technique over mitral valve replacement for severe primary mitral regurgitation (MR), where a durable and successful repair is expected [2,3]. Additionally, where ventricular function is preserved and the patient is asymptomatic, the aforementioned guidelines stipulate that surgery should only take place in centres specialising in valve surgery, where the expectation of durable repair is high and mortality is low [2, 3]. A strong inverse relationship is known to exist for many procedures between surgeon volume and mortality [4] and hospital volume and mortality [5]. Similar findings have been observed in studies based on United States (US) data [6-9]. However, no Australasian study has examined the relationship between surgeon and hospital volume and mitral valve repair rates and mortality for degenerative MR.

It is therefore the purpose of this investigation to ascertain whether, in an Australian cohort, a relationship exists between hospital and surgeon procedural volume and mitral valve repair rates and 30-day mortality. Excluding other pathologies such as valve stenosis which adversely affect propensity for repair, the authors 
sought to identify the effect of this "modifiable" variable of procedural volume on patient outcomes. In our low-density Australian population the feasibility of establishing "heart valve centres" may be limited. Nonetheless, we sought to come up with a recommendation regarding annual procedural volume for hospitals and surgeons to maximise repair rates and minimise operative mortality.

\section{Materials and Methods}

\section{Data source}

The Australian and New Zealand Society of Cardiac and Thoracic Surgeons (ANZSCTS) systematically collects and monitors data related to cardiac surgical procedures in Australia and New Zealand through the ANZSCTS Database. It currently has 41 participating government-funded and private hospitals, of which until recently all were in Australia; the remaining 31 cardiac surgical institutions do not contribute [10]. These sites collectively perform approximately 14,000 cardiac operations per year [10]. A Data Definition Manual is available via the ANZSCTS Database website [11].

\section{Study design}

This study was approved by the Monash Health Human Resource Ethics Committee (reference number HREC/43701/MonH-2018-67577). This was a retrospective cohort study, limited to adults (aged [?]18) who underwent either mitral valve repair or replacement for degenerative mitral valve disease, as identified in the ANZSCTS Database from January 12008 to December 31 2017. The data identifier for mitral procedure type was Item \#91.0, codes 3 'replacement', 4 'repair or reconstruction with annuloplasty' and 5 'repair or reconstruction without annuloplasty'. The aetiology of mitral disease, Item \#91.10, was narrowed by code 5 'myxomatous degeneration', encompassing the spectrum of fibroelastic deficiency through to Barlow's disease. There were no exclusions. Concomitant coronary artery bypass grafting (CABG), non-CABG major cardiac surgery and surgery for atrial arrhythmias were included as binary variables (see 'Variables' below).

\section{Measured outcomes}

The primary outcome assessed was: is surgeon or hospital volume associated with the rate of mitral valve repair in myxomatous degeneration. A secondary outcome evaluated was: is 30-day mortality associated with mitral valve repair in myxomatous degeneration, taking into account surgeon and or hospital volume.

\section{Variables}

Annual mean volume for surgeon and hospitals were determined by dividing the total number of procedures by the number of years for which the individual surgeon or hospital had data collected. Surgeon and hospital annual procedural volume were then categorised as [?] 5, 5.1-10, 10.1-20, and 20.1-45, for surgeons and [?] $10,10.1-20,20.1-30$, and 30.1-75, for hospitals. We chose these points rather than quartiles as these gave arbitrary cut-off points with minimal clinical applicability.

Regarding procedural variables, we defined ' $\mathrm{CABG}$ ' as any concomitant coronary artery bypass, irrespective of number of grafts. We defined any 'major concomitant cardiac surgery' as any other valve surgery, left ventricular reconstruction, ventricular septal defect repair, left ventricular outflow tract myectomy, pericardiectomy, pulmonary thromboendarterectomy or embolectomy, resection of cardiac tumour, any aortic procedure and any other surgery for congenital cardiac disease (excluding atrial septal defect). A third category of 'atrial arrhythmia surgery' was utilised; the specific lesion set was not captured. Excluded from these three categories of concomitant procedures, and therefore not defined as variables for logistic regression analysis, were atrial septal defect closures, minor cardiac procedures such as insertion of a left ventricular pacing lead, and exclusion/excision of the left atrial appendage, as well as thoracic operations. These were not deemed to add significant risk or alteration to surgical decision-making with respect to the mitral valve surgery. Procedural urgency was defined as per the ANZSCTS Database as elective, urgent or emergency; there were no salvage procedures in this cohort [11].

\section{Statistical analyses}


Normally distributed continuous data was presented as mean \pm standard deviation and analysed using ANOVA, whereas skewed data was presented as median (interquartile range) and analysed using Wilcoxon rank-sum test. Categorical data was presented as number (percent) and analysed using either chi-square or Fisher's exact test as appropriate.

To take into account the clustering of patients under surgeon, univariate and multivariate mixed effects logistic regression were performed to examine the associations between characteristics and the binary outcomes. As some surgeons operated in multiple hospitals the clustering of hospitals was not included in the models.

Due to the low incidence of 30-day mortality, the multivariate mortality analysis was constrained by the number of variables which could be included in the model. Surgeon and hospital volume were analysed as continuous rather than categorical variables. Age and gender were selected as a priori variables in addition to mitral valve repair.

The odds ratios (OR) for continuous variables including age and Body Mass Index (BMI) were presented in increments of 5 units. Stata version 14.2 was used for analysis. Statistical tests were carried out at a 2-sided 0.05 level of significance.

\section{Results}

Between January 2008 to December 2017, a total of 40 hospitals and 124 surgeons performed 4,970 mitral valve operations for degenerative MR, with 3,405 (68.5\%) being repairs.

The repair group tended to be younger, more likely to be male, elective, and associated with increased surgeon and hospital volume. The repair group was less likely to be associated with urgent surgery, infective endocarditis, reduced LVEF, increased NYHA and other comorbidities such as diabetes (See Table 1). On multivariate analyses, hospital and surgeon remained associated with repair after adjustment for other confounders (See Table 2).

Data regarding 30-day mortality was available for $99.9 \%$ of patients. The all-cause mortality rate was $1.67 \%$ overall and $1.18 \%$ for repair and $2.75 \%$ for replacement (OR of unadjusted 30-day mortality of repair compared to replacement $0.42,95 \%$. CI $0.27-0.65, \mathrm{p}<0.01)$. On univariate analysis, the variables associated with 30-day mortality were increased age, pre-operative dialysis, peripheral vascular disease, significant coronary disease, congestive heart failure, increase NYHA Class, moderate or severely impaired left ventricle (EF [?]45\%), non-elective status, concomitant major cardiac surgery or CABG, and mitral valve replacement (see Table 3). The median hospital volume (kept continuous due to small numbers) was lower (21.5 per year) for those that died compared to alive at 30-days (24.3 per year), which was no longer significant after adjustment for age and gender (see Table 4).

\section{Discussion}

By focussing on degenerative MR for which repair is the gold-standard, excluding operations for mitral stenosis and non-degenerative MR, the cohort size was reduced. To increase the cohort size, we chose to analyse all procedures for degenerative MR rather than only isolated procedures. This is in contrast to the four previously reported comparable US studies which structured their analyses differently, each of which varied both in inclusion of different aetiologies of mitral valve disease and of concomitant procedures [6-9].

Our study identified that only two surgeons in Australia in the evaluated time period performed close to 40 procedures annually, whereas the top four North American surgeons by volume were performing more than 100 isolated mitral valve surgeries annually [7], albeit "only a handful" of North American surgeons perform more than 50 annually [12]. Despite this difference in volume, Australian surgeons have achieved reasonable results. The overall repair rate was $68.5 \%$, or $73.4 \%$ for isolated mitral valve procedures (+atrial arrhythmia surgery). The comparable STS data revealed an $82.5 \%$ repair rate for isolated mitral valve surgery for "degenerative leaflet prolapse," though no repair rate for mitral valve surgeries with concomitant procedures was available [13]. The Australian 30-day mortality outcomes were excellent, with an overall $1.67 \%$ 30-day mortality rate (unadjusted) for mitral valve procedures including concomitant CABG or other 
major cardiac surgery, and $0.9 \%$ for isolated mitral valve procedures (+- atrial arrhythmia surgery). The comparable STS mortality rate for isolated mitral valve procedures for degenerative MR was $1.2 \%$, albeit risk profile could not be compared [13], and no US mortality rate was available for surgery for degenerative MR with concomitant procedures.

Of note, almost $50 \%(2,471$ of 4,965) of operations were performed by surgeons only performing up to 10 procedures per year. This is similar to North America, where STS data suggests that the average surgeon performs fewer than 10 mitral valve surgeries per year [12]. This is problematic, as it may indicate that there is a proportion of patients undergoing replacement for potentially reparable pathology. Whereas Bridgewater et al's United Kingdom group attempted to establish "best practice standards" for mitral surgery, recommending an individual surgeon threshold of more than 25 annual procedures and hospital threshold of 50 [14], Bonow et al acknowledge that these volumes would be "difficult to meet at many centers [sic] [12]." However, a threshold of 10 procedures per surgeon or hospital, at a minimum, is likely more achievable.

\section{Limitations}

Our study has several limitations. Firstly, the ANZSCTS Database collects different data points to major international registries such as the STS and the European database utilised for creating the EuroSCORE II. In contrast to the STS database, procedure type is categorised differently, and ethnicity data, previous mediastinal radiation, cancer and chronic liver disease are not captured [15]. Unlike EuroSCORE II, ANZSCTS does not capture pulmonary hypertension as a risk factor [16]. Consequently, we could not correctly calculate the EuroSCORE II nor STS score for our patients, limiting direct comparability of risk profiles to international data.

Secondly, by focussing on the most recent decade of procedures to fit contemporaneous standards of best practice, we have limited our follow-up period to 30 days. This limits the capacity to assess any longerterm outcome difference between patients who underwent repair versus replacement. Nonetheless, this study is founded on the a priori assumption that repair is preferable to replacement with respect to long-term mortality. Additionally, a 30-day follow-up does not allow for assessment of reoperation rate or repair durability, relevant to both the quality of repair and the durability of replacement prostheses. As directed by the European guidelines for management of chronic primary MR, "achieving a durable valve repair is essential [2]l;" a competent valve intraoperatively or at 30 days does not necessarily equate with durability.

Finally, the presence of only 83 30-day mortalities limited the capacity to assess contributing variables, and in particular to assess for the effect of procedural volume. In comparison, Vassileva et al's similar study using Medicare Provider Analysis and Review data from the US analysed a cohort of 125,079 patients aged 65 and over; a hospital mortality rate of $10.7 \%$ equated to more than 12,000 patients [8]. This much larger cohort and much higher mortality rate facilitated assessment of impact of procedural volume on mortality, which we were unable to achieve to statistical significance.

\section{Conclusion}

This study evaluated characteristics associated with mitral valve repair rates and 30-day mortality for degenerative MR, focussing on the impact of procedural caseload. It conclusively demonstrates that within the Australian cohort, annual surgeon caseload was strongly associated with repair rate, with hospital caseload less strongly but also associated with higher repair rate. Surgeons with an annual volume of $>20$ demonstrated the highest odds of repair, approximately four times that of surgeons performing [?]5/year. Repair rate for these higher volume surgeons was approximately $80 \%$, compared with approximately $57 \%$ in the lowest volume category. A threshold of $>10$ procedures/year per surgeon was defined as a statistically significant cut-off to improve the odds of repair, though above this threshold, greater volume was generally associated with higher odds of repair, particularly $>20$ /annum. For hospitals, the same threshold of $>10$ procedures was statistically significant for improving the odds of repair, though the caseload above this cut-off did not appear to matter. Repair rate for hospital caseload [?]10/annum was $52 \%$, climbing to approximately $71 \%$ for hospitals above this threshold. This study, therefore, demonstrated that to maximise the likelihood 
of repair for degenerative MR, individual hospitals and surgeons should be performing more than 10 such procedures annually.

Additionally, this study demonstrated that in the Australian cohort, wherein unadjusted 30-day mortality rate for repair was $1.18 \%$ and $2.75 \%$ for replacement, mitral valve repair conferred a significant 30-day survival advantage when adjusting for age, gender and procedural volume. Although mortality was not significantly affected by surgeon and hospital procedural volume, unadjusted data nonetheless suggests that higher volumes may reduce mortality rates.

Finally, this data provided support for the consideration of mitral valve surgery as a subspecialty within cardiac surgery. Both for higher-complexity degenerative pathology, as well as for the asymptomatic patient in whom the guideline expected likelihood of durable repair must be high or in excess of $95 \%[2,3]$ such patients should be considered for referral to higher volume surgeons. This may therefore require surgeon inter-referral, as well as encouragement of such referral patterns by Cardiologists to mitral subspecialist surgeons.

\section{Acknowledgements}

The Australian and New Zealand Society of Cardiac and Thoracic Surgeons (ANZSCTS) National Cardiac Surgery Database Program is funded by the Department of Health, Victoria, the Health Administration Corporation (GMCT) and the Clinical Excellence Commission (CEC), NSW, and funding from individual Units. ANZSCTS Research activities are supported through a National Health and Medical Research Council Senior Research Fellowship and Program Grant awarded to C.M. Reid.

\section{Funding}

This study was supported by funding from the Department of Surgery (School of Clinical Sciences at Monash Health), Monash University

\section{Conflict of interest}

None declared

\section{Author contributions}

Stephanie Wayne: Drafting article, concept/design, data analysis/interpretation

Catherine Martin: Statistics, data analysis/interpretation

Julian Smith: Critical revision of article, securing funding

Aubrey Almeida: Concept/design, critical revision of article, approval of article

\section{References}

[1] Nishimura RA, Vahanian A, Eleid MF, Mack MJ. Mitral valve disease - current management and future challenges. Lancet 2016 Mar 26;387(10025):1324-34

[2] Falk V, Baumgartner H, Bax JJ, De Bonis M, Hamm C, Holm PJet al . 2017 ESC/EACTS Guidelines for the management of valvular heart disease. Eur J Cardiothorac Surg 2017 Oct 1;52(4):616-664

[3] Nishimura RA, Otto CM, Bonow RO, Carabello BA, Erwin JP, Guyton RA et al. 2014 AHA/ACC guideline for the management of patients with valvular heart disease: executive summary: a report of the American College of Cardiology/American Heart Association Task Force on Practice Guidelines. J Am Coll Cardiol 2014 Jun 10;63(22):2438-88

[4] Birkmeyer JD, Stukel TA, Siewers AE, Goodney PP, Wennberg DE, Lucas FL. Surgeon volume and operative mortality in the United States. N Engl J Med 2003 Nov 27;349(22):2117-27

[5] Reames BN, Ghaferi AA, Birkmeyer JD, Dimick JB. Hospital volume and operative mortality in the modern era. Ann Surg 2014 Aug;260(2):244-51 
[6] Kilic A, Shah AS, Conte JV, Baumgartner WA, Yuh DD. Operative outcomes in mitral valve surgery: combined effect of surgeon and hospital volume in a population-based analysis. J Thorac Cardiovasc Surg 2013 Sep;146(3)638-46

[7] Bolling SF, Li S, O’Brien SM, Brennan JM, Prager RL, Gammie JS. Predictors of mitral valve repair: clinical and surgeon factors. Ann Thorac Surg 2010 Dec;90(6):1904-11

[8] Vassileva CM, McNeely C, Spertus J, Markwell S, Hazelrigg S. Hospital volume, mitral repair rates, and mortality in mitral valve surgery in the elderly: an analysis of US hospitals treating Medicare fee-for-service patients. J Thorac Cardiovasc Surg 2015 Mar;149(3):762-8

[9] LaPar DJ, Ailawadi G, Isbell JM, Crosby IK, Kern JA, Rich JBet al . Mitral valve repair rates correlate with surgeon and institutional experience. J Thorac Cardiovasc Surg 2014 Sep;148(3):995-1003

[10] Australian and New Zealand Society of Cardiac and Thoracic Surgeons (ANZSCTS). About the Database (internet). ANZSCTS; 2019. Accessible athttps://anzscts.org/database/about/\#DDM

[11] Australian and New Zealand Society of Cardiac and Thoracic Surgeons (ANZSCTS). Data Definitions Manual Version 4 (internet). ANZSCTS; 2017. Available from: https://anzscts.org/database/about/\#DDM

[12] Bonow RO, Adams DH. The time has come to define centers of excellence in mitral valve repair. J Am Coll Cardiol 2016 Feb 9;67(5):499-501

[13] Gammie JS, Chikwe J, Badhwar V, Thibault DP, Vemulapalli S, Thourani VH et al . Isolated Mitral Valve Surgery: The Society of Thoracic Surgeons Adult Cardiac Surgery Database Analysis. Ann Thorac Surg 2018 Sep;106(3):716-727

[14] Bridgewater B, Hooper T, Munsch C, Hunter S, von Oppell U, Livesey S et al . Mitral repair best practice: proposed standards. Heart 2006 Jul;92(7):939-44

[15] Society of Thoracic Surgeons (STS). Online STS Risk Calculator (internet). STS; 2019. Accessible at:http://riskcalc.sts.org/stswebriskcalc/calculate

[16] EuroSCORE Study Group. EuroSCORE Interactive Calculator (internet). EuroSCORE Study Group; 2011. Accessible at:http://www.euroscore.org/calc.html

\section{Hosted file}

Article for JCardSurg Figure 1.docx available at https://authorea.com/users/350953/articles/ 475644-mitral-valve-repair-rates-in-degenerative-mitral-valve-disease-correlate-withsurgeon-and-hospital-procedural-volume

\section{Hosted file}

Article for JCardSurg Figure 2.docx available at https://authorea.com/users/350953/articles/ 475644-mitral-valve-repair-rates-in-degenerative-mitral-valve-disease-correlate-withsurgeon-and-hospital-procedural-volume

\section{Hosted file}

Article for JCardSurg Table 1.docx available at https://authorea.com/users/350953/articles/ 475644-mitral-valve-repair-rates-in-degenerative-mitral-valve-disease-correlate-withsurgeon-and-hospital-procedural-volume

\section{Hosted file}

Article for JCardSurg Table 2.docx available at https://authorea.com/users/350953/articles/ 475644-mitral-valve-repair-rates-in-degenerative-mitral-valve-disease-correlate-withsurgeon-and-hospital-procedural-volume

\section{Hosted file}


Article for JCardSurg Table 3.docx available at https://authorea.com/users/350953/articles/ 475644-mitral-valve-repair-rates-in-degenerative-mitral-valve-disease-correlate-withsurgeon-and-hospital-procedural-volume

\section{Hosted file}

Article for JCardSurg Table 4.docx available at https://authorea.com/users/350953/articles/ 475644-mitral-valve-repair-rates-in-degenerative-mitral-valve-disease-correlate-withsurgeon-and-hospital-procedural-volume 